\title{
COVID-19 variants that escape vaccine immunity: Global and Indian context-are more vaccines needed?
}

\author{
Bijayeeta Deb, Ramya Vilvadrinath and Suchi Goel* \\ Indian Institute of Science Education and Research (IISER) Tirupati, Tirupati, India \\ *Corresponding author (Email, suchi@iisertirupati.ac.in)
}

MS received 23 July 2021; accepted 28 September 2021

\begin{abstract}
The COVID-19 pandemic that emerged around December 2019 claimed millions of lives. For vaccine development, S protein on viral envelope that binds to ACE2 receptor on cells for entry was identified as vaccine candidate. S protein consists of Receptor Binding Motif (RBM) in the S1 subunit followed by the S2 subunit with an intermediate furin cleavage site. A stabilized version of S protein with 2 proline residues was used as antigen. Overall, most vaccines exhibited efficacy between 80 and 95\%. However, being a RNA virus that is prone to mutations along with selection pressure on S protein and frequent use of convalescent plasma led to evolution of variants. These variants are responsible for multiple waves of infection observed globally. In our review, we discuss current data on vaccines and its efficacy in neutralizing SARS-CoV-2 from Wuhan and its variants. Further, our docked mutations observed in variants on the ACE2-S complex cryo-EM structure show that mostly the $\mathrm{S} 1$ domain is under selection pressure where major mutations occur in the $\mathrm{N}$ terminal domain (NTD), RBM and junction near S1-S2 subunit. Therefore, this review would be a reference for development of new candidate antigen(s) with better efficacy against variants.
\end{abstract}

Keywords. Covid-19; SARS-CoV-2; vaccines; variants

\section{Introduction}

With the identification of SARS-CoV-2 responsible for COVID-19 in 2019, the virus has been under constant surveillance and discussion with regards to its origin, evolution, transmission, mutation, disease symptoms as well as its effective mechanism of hijacking the host immune system. Using genomic and taxonomic evidence, it has been clearly proved that it belongs to order Nidovirales, family Coronaviridae, subfamily Coronavirinae, genus Betacoronavirus, and subgenus Sarbecovirus. Similar to other viruses of the genus betacoronavirus, SARS-CoV-2 is an enveloped virus with positive-sense, ssRNA genome of $\sim 30 \mathrm{~kb}$ size and it is $\sim 80 \%$ homologous to SARS and $\sim 50 \%$ to MERS (Kim et al. 2020; Zhou et al. 2020).

This article is part of the Topical Collection: COVID-19: Disease Biology \& Intervention.
The SARS-CoV-2 genome consists of non-segmented RNA and encodes 13-15 open reading frames (ORFs). The RNA encodes for 7096 amino acid long polypeptide that is cleaved by viral proteinases to yield two non-structural proteins replicase and protease at the $5^{\prime}$ end. The remaining polypeptide at the $3^{\prime}$ end encodes for structural proteins like Spike (S), Envelope (E), Membrane (M) and Nucleocapsid (N) protein, and 5 accessory proteins (ORF3, ORF4a, ORF4b, ORF5 and ORF8) important for formation of capsid and envelope of the virus (Kim et al. 2020).

Being from the betacoronavirus genus, the virus originated from bats and was thought to be transmitted to humans with the help of an intermediate animal. However, even after a year since the identification of this virus, none of the studies have been able to provide experimental proof about the identity of the intermediate host. Along with this, though the $\mathrm{S}$ protein from SARS-CoV-1 and SARS-CoV-2 are 75\% similar, the latter consists of a furin cleavage site that is a signature 
of Influenza virus (Gralinski and Menachery 2020; Zhou et al. 2020). The absence of conclusive evidence of its mode of reaching humans and the presence of a furin cleavage site in $\mathrm{S}$ protein raises the concern of alternate theory of lab leak from a lab in Wuhan, China. However, this theory still needs to be scientifically established. It is also important to note that this furin cleavage site is from the Influenza virus that is known for its high transmission rates (Andersen et al. 2020). This suggests that the presence of this site might be the reason why SARS-CoV-2 has higher transmission rates as compared to previous SARS and MERS epidemics that were far more contained.

\section{What should be a vaccine candidate?}

For an antigen to be a successful vaccine candidate, it should be able to block the first step of infection, that is the entry of the virus into the cells. The ACE2 receptors present abundantly on the epithelial cell surface of the oral mucosa, human lungs and small intestine act as receptors for $\mathrm{S}$ protein on the viral envelope. The initial interaction between the SARS-CoV-2 and host occurs at the surface of the epithelium, with the attachment of $\mathrm{S}$ protein to the ACE2 receptor. The S protein consists of two subunits S1 and S2 that are cleaved by a protease at the furin cleavage site. After cleavage, S1 contains the Receptor Binding domain (RBD) that binds to ACE2 while S2 subunit mediates fusion of viral envelope to cells. This fusion leads to release of the viral genome that is followed by replication and proliferation of virus inside the cells (Pallesen et al. 2017). This hijacking of host cell machinery and immune system for its multiplication is responsible for the pathogenic symptoms associated with SARS-CoV2 like lung inflammation, pneumonia and even death.

Thus in order to stop the infection and pathogenesis of SARS-CoV-2, the vaccine efforts should focus on abrogating the binding of $\mathrm{S}$ protein to the ACE2 receptor. The S protein belongs to Class I fusion proteins, and its cryo-EM structure revealed that it exists in trimeric form similar to the $\mathrm{S}$ protein from MERS virus (Wrapp et al. 2020; Pallesen et al. 2017). However, in the case of SARS-CoV-2, one of the RBD domains was found to be 'up' while the other two were in 'down' position. The binding of RBD in 'up' position with ACE2, induces the movement of remaining RBDs to 'up' position, forming a multimeric binding interaction. After binding, S1 is shed by a protease, while S2 forms a prehairpin intermediate, followed by fusion and entry of the virus into the host cell (Wrapp et al. 2020).

Though the structure of S protein identified the mode of binding and its subsequent establishment of infection in host cells, in order to design $\mathrm{S}$ protein as a successful antigen for vaccine, it was necessary to obtain detailed information on the S protein-ACE2 interaction. Therefore, efforts were made for structure based antigen design by solving the structure of RBD in complex with ACE2 using cryo-EM. The structure was of high resolution $(3.5 \AA)$ and revealed that the interacting site was enriched in tyrosines and is hydrophilic in nature with 13 hydrogen bonds and 2 salt bridges. Although the structure is similar to the RBD of SARS-CoV-1, there is an additional interaction site (K417) outside the RBD domain that interacts with D30 of ACE2. Further there is presence of positively charged patch contributed by K417 that might be responsible for the higher binding affinity of SARSCoV-2 RBD to ACE2 than SARS-CoV-1 RBD. It is interesting to note that although the binding mode and structure is similar between these two viruses, the neutralization antibodies of SARS-CoV-1 did not neutralize SARS-CoV-2, suggesting its implications on variants of SARS-CoV-2 (Lan et al. 2020; Xu et al. 2021).

\section{Vaccine efforts}

The structure of the S protein and S-ACE2 complex was critical for vaccine efforts and accelerated the vaccine development process. However, the major challenge was to design a construct that would render the protein stable in vivo. Additionally, the presence of a protease target site made it amenable to degradation. In this aspect, the studies from MERS virus were quite helpful where it was observed that incorporation of 2 proline residues stabilized the protein (Pallesen et al. 2017). Similar approach was taken for S protein of SARS-CoV-2, where $\mathrm{S}$ protein was expressed from amino acids 1-1208 and proline was introduced at amino acid positions 986 and 987. Further furin cleavage site (RRAR) was mutated to 'GSAS' (Wrapp et al. 2020). This structure based design proved successful and is the backbone of the vaccine constructs from various manufacturers like Moderna (mRNA1273), Pfizer (BNT162b2), Novavax (NVX-CoV2373) and Janssen (Ad26.COV2.S). Different manufacturers decided to use different strategies for delivery of 


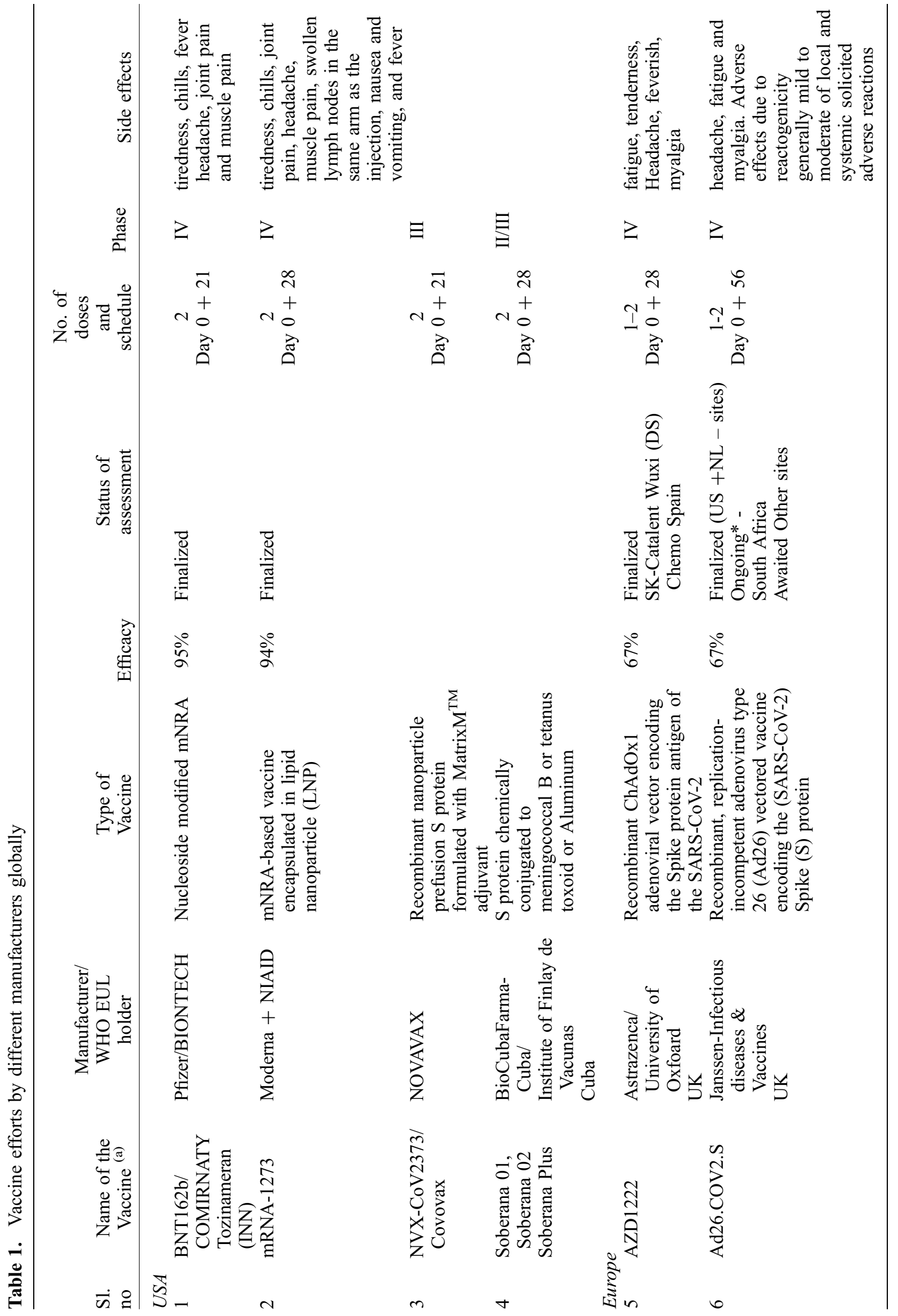




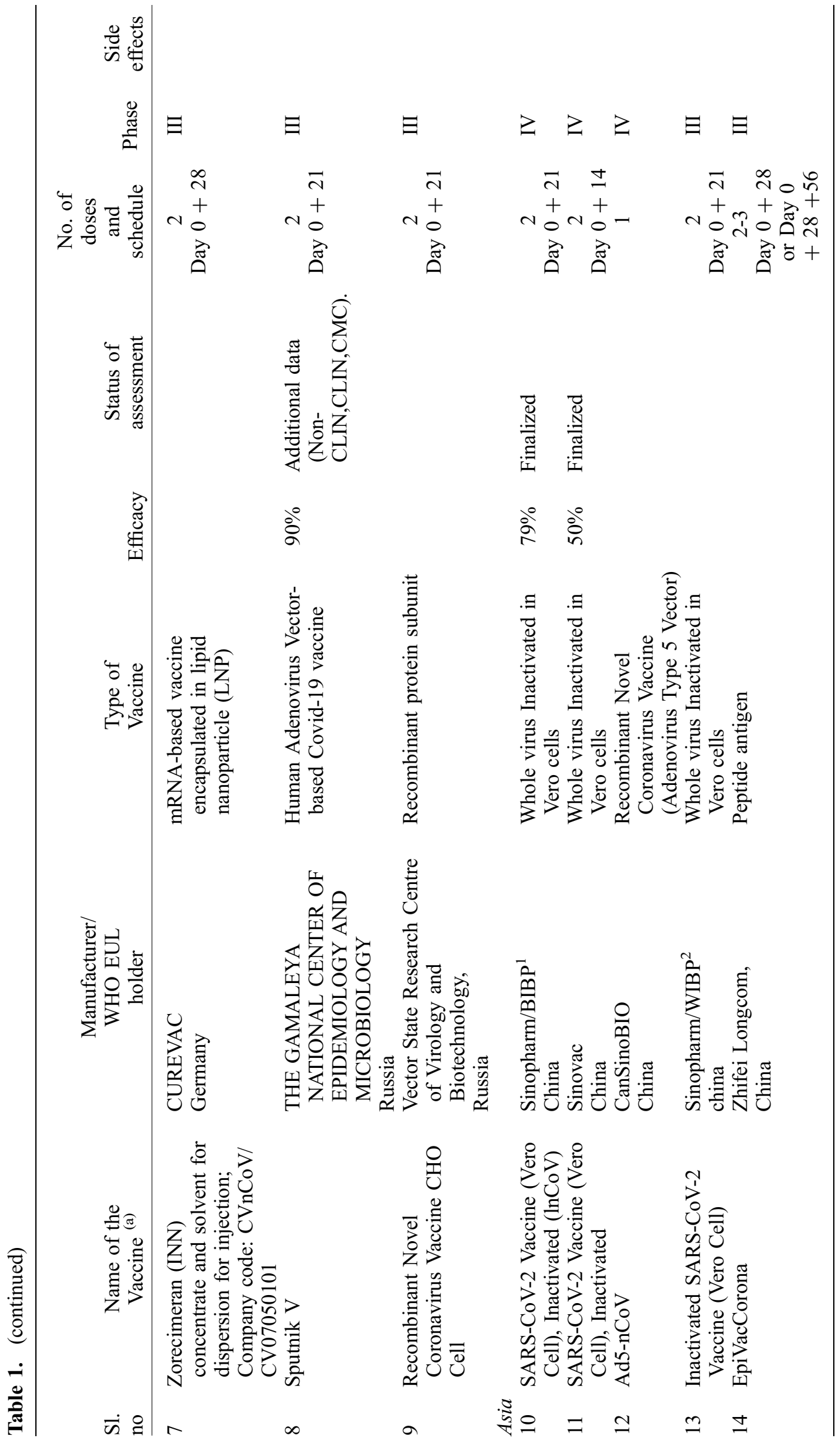




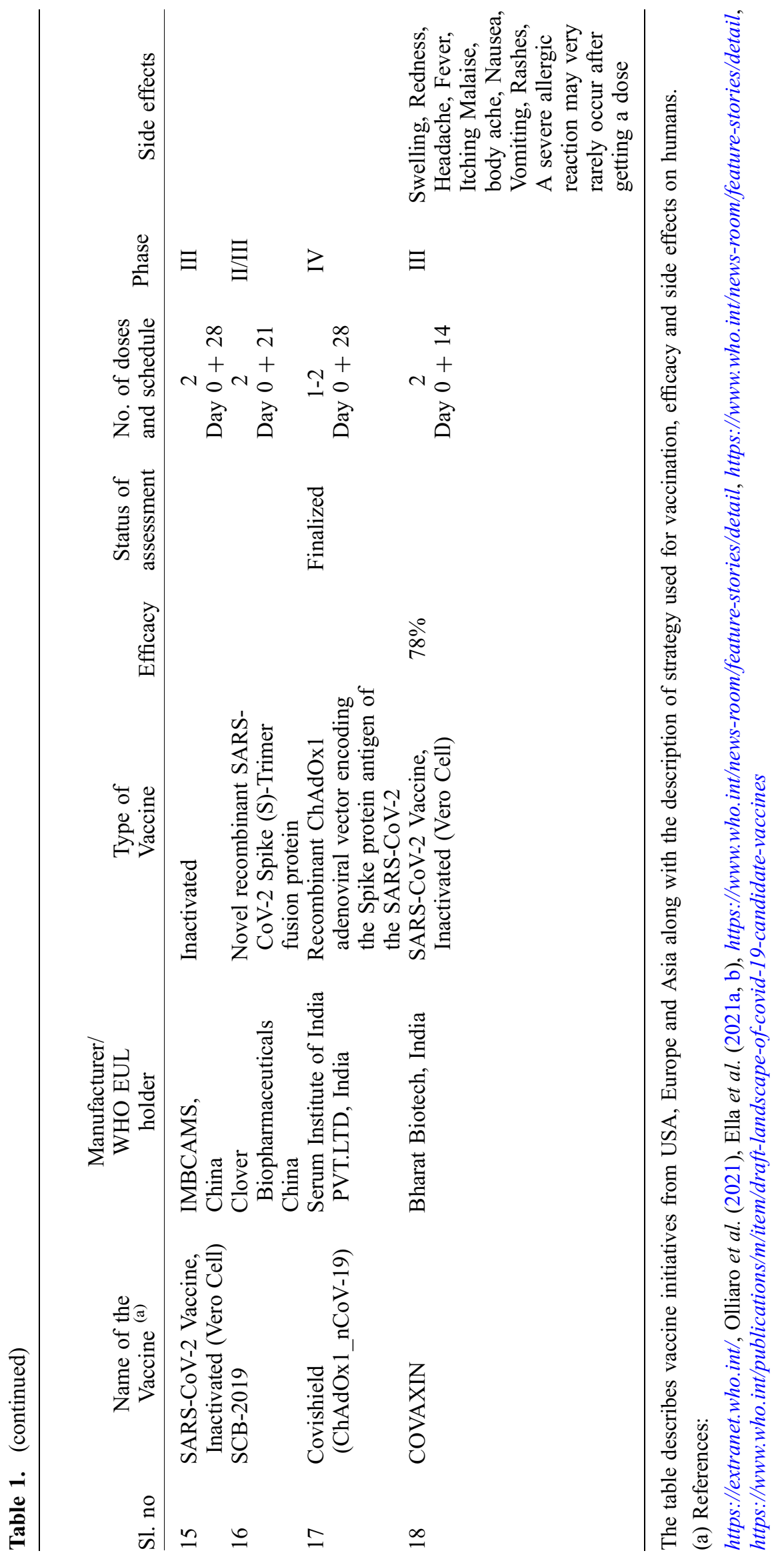



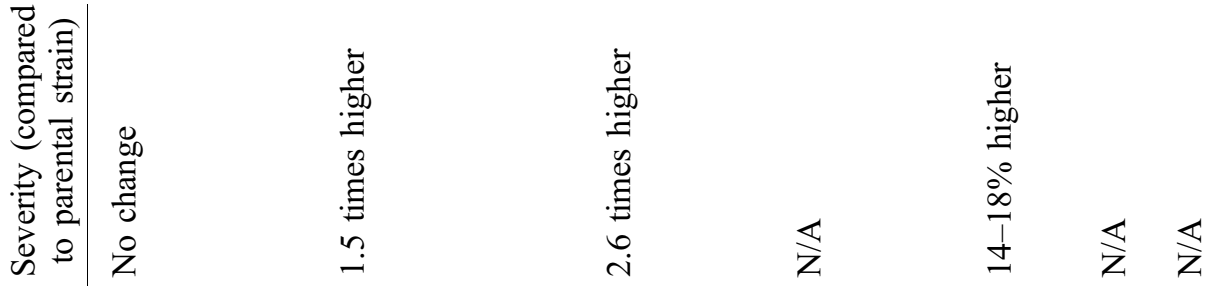

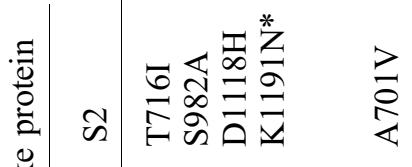

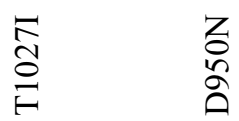

$\begin{array}{ll}\stackrel{1}{6} & \infty \\ \equiv & \infty \\ \supset & \infty \\ 1 & \infty\end{array}$

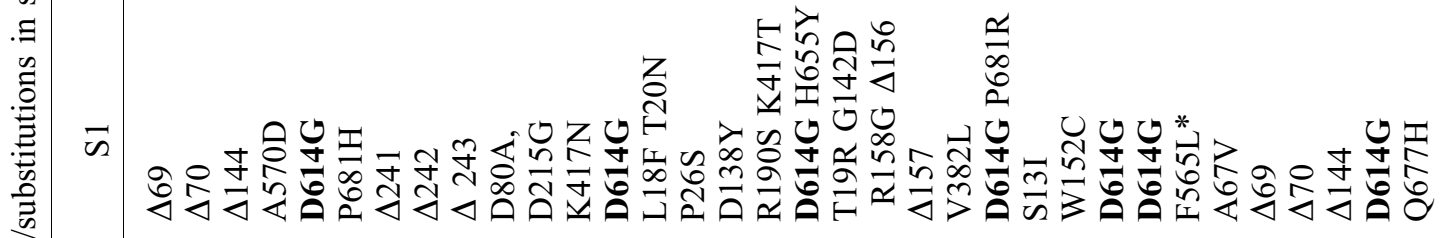

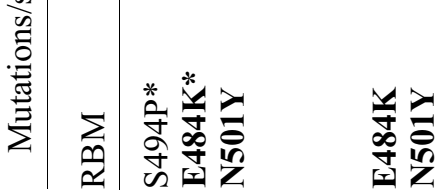

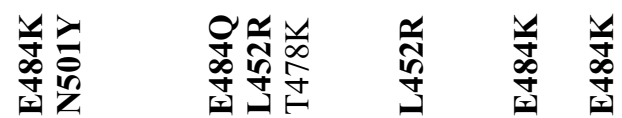

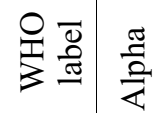

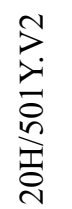

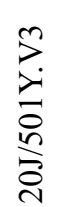

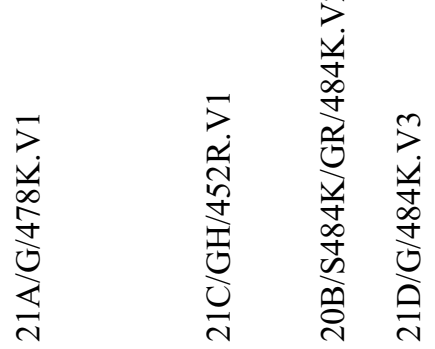

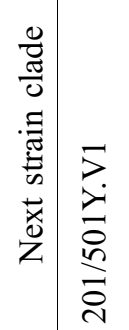

ஸ্ঠ்

$\underset{\Xi}{\overparen{\Xi}}$

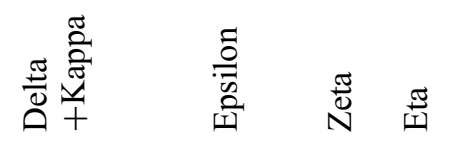

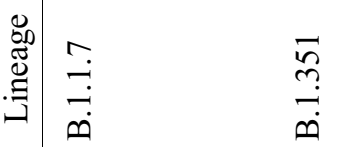

กิก

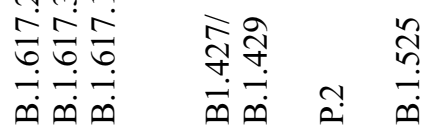

త్త

䨌

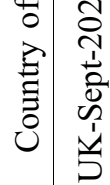

a

त̄

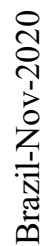

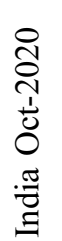

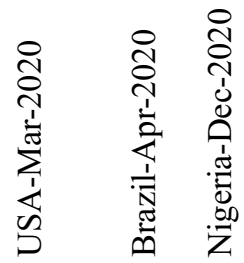

$\stackrel{\Xi}{\omega}$
$\dot{\infty}$

N

$m$

n 6 - 
COVID-19 variants that escape vaccine immunity

Page 7 of $16 \quad 112$

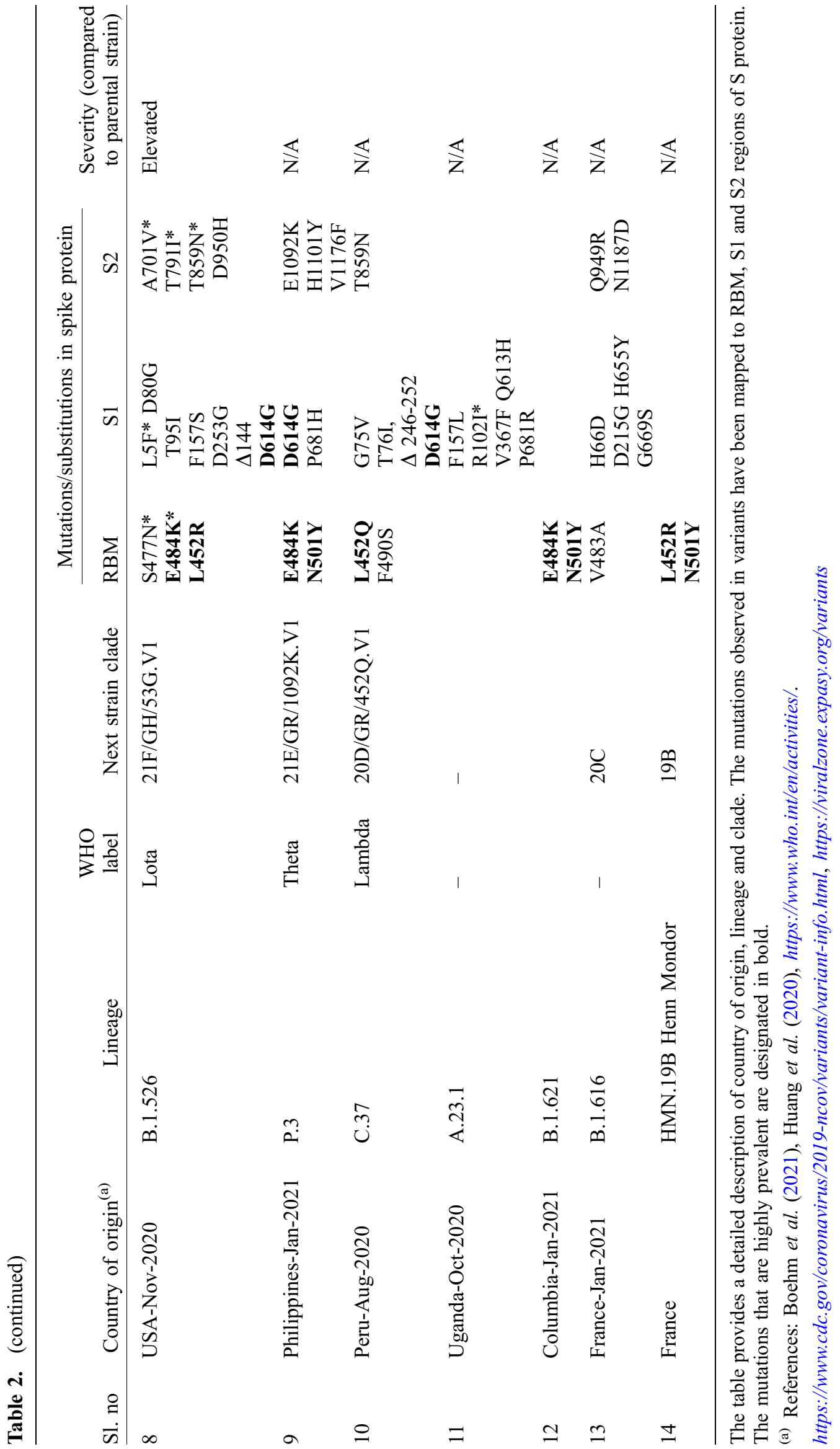


antigen but all of their constructs use $\mathrm{S}$ protein with 2 proline substitutions.

In the quest to further improve the design of vaccines that have enhanced stability, Hsieh $\mathrm{C}$ et al., also came up with various changes in the $\mathrm{S}$ protein such as disulfide bridges, addition of prolines, salt bridges and cavity filling approach. Of all these, cavity filling and proline approach worked better and now combining all these works, they have come up with a combinatorial antigen that has the advantage of stability with increased expression capacity (Hsieh et al. 2020).

Although this combinatorial antigen still needs to be tested, in order to limit the number of COVID19 infections and mortality, various companies already initiated the efforts for vaccines by bypassing the traditional steps of vaccine development (Deb et al. 2020). Most of the companies in different countries have taken the approach of using $\mathrm{S}$ protein as the antigen with inactivated replication deficient transfer vector. Moderna and Pfizer used the mRNA approach, and they were among the initial companies that started vaccinations in the USA. Russia developed Sputnik $\mathrm{V}$ and India developed Covishield in collaboration with Oxford University, UK. Most of the vaccines have been estimated to have $80-95 \%$ efficacy against SARSCoV-2 (table 1). Although most of the candidates were considered safe in Phase I trials, ongoing vaccinations demonstrate the side effects of these vaccines. The vaccine from Janssen was stopped due to cerebral venous thrombosis (CVST) with thrombocytopenia after vaccinations (Sadoff et al. 2021). Covishield was also associated with blood clotting (https://www.publichealth.hscni.net/publicat ions/blood-clotting-following-covid-19-vaccinationinformation-health-professionals) while Pfizer vaccine was found to have long term implications in heart inflammation (Marshall et al. 2021).

In contrast to the above mentioned manufacturers, Sinopharm from China and Bharat Biotech in collaboration with Indian Council of Medical Research (ICMR) India, applied the conventional strategy of using inactivated virus as the antigen. Both of them used $\beta$-propionolactone for inactivation of the virus (Wang et al. 2020; Ella et al. 2021a, b), though the viral strains were different (Ganneru et al. 2021). Based on the press release from Bharat Biotech, Covaxin is currently predicted to be successful against clearing infection with $78 \%$ efficacy, with minimal side effects (Ella et al. 2021a, b; table 1). However, it is still early to conclude about its effects on the population and the ongoing Phase III trials will provide more information.
Figure 1. Analysis of mutations in the $\mathrm{S}$ region of SARSCoV-2 variants. (A) Sequence alignment of $\mathrm{S}$ protein of variants sequenced till date. The mutations are mapped to the RBM, S1 and S2 region of the protein. (B) Docking of mutations observed in S protein of variants in complex with ACE2. The S protein-ACE2 complex cryo-EM structure (PDB ID: 7DF4; Xu et al. 2021) is used for mapping mutations.

\section{Response of humans to vaccination}

Due to high infection rates and mortality, and with a large proportion of the population falling in the vulnerable category, vaccine initiatives started quite early in the USA and UK. Pfizer and Moderna in the USA and Covishield in the UK administered vaccines as two doses separated by several weeks. The Pfizer (BNT162b2) vaccination led to generation of a strong antibody response and the response generated after the 2nd dose was comparable with that of the people who contracted COVID-19 and were given the $1^{\text {st }}$ dose of vaccine (Ebinger et al. 2021). However, the studies with the same vaccine showed that the antibody response decreased by 6 weeks after the 2nd dose, suggesting that the immune protection is not long lasting and tends to fade away eventually. Also, older males showed decreased vaccine efficacy (Naaber et al. 2021). On a positive note, recent data from vaccinations in Israel conveyed that BNT162b2 was able to reduce hospitalizations and severe disease by $87 \%$ and $92 \%$ respectively and prevented $94 \%$ of the symptomatic COVID-19 cases. Similar results were obtained from vaccinations performed in Scotland with Covishield from Oxford university, where total effectiveness was around 81\% (Dagan et al. 2021).

For India, due to its large population, it would have been difficult to depend on foreign sources for vaccinations. As India already had expertise and facilities in making vaccines for many diseases, it successfully developed its own vaccines. Initial efforts were majorly from Covishield manufactured by Serum Institute in collaboration with Oxford University and Covaxin from Bharat Biotech in collaboration with ICMR. At first, during vaccine development, it was not clear which strategy would be successful, so India focused on Covishield that used an adenoviral vector delivery method and Covaxin which relied on an inactivated virus method. Both of the antigens showed a high seropositivity rate of $79.3 \%$ after the first dose of vaccine and no differences were observed with age and gender. Also, Covishield showed better response to anti-Spike protein as compared to Covaxin $(86.8 \%$ vs $43.8 \%)$ but 
A

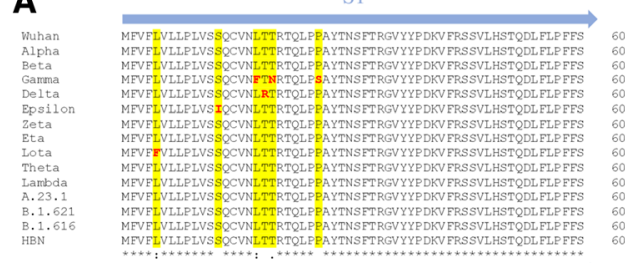

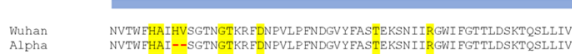

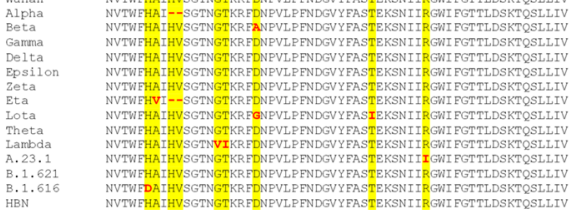

B. 1.616
HBN

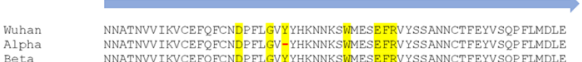

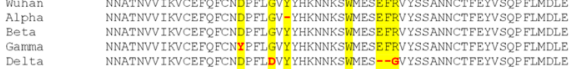

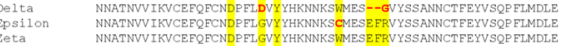

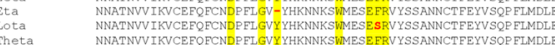

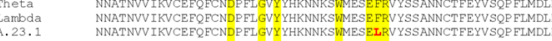

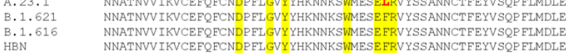
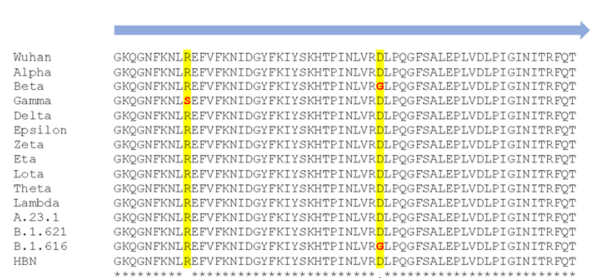

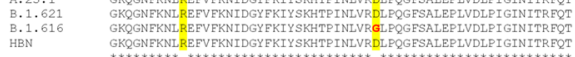

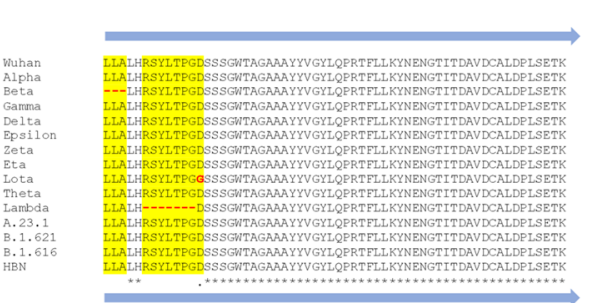

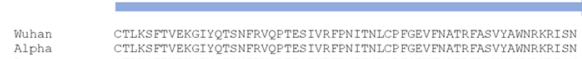

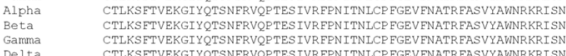

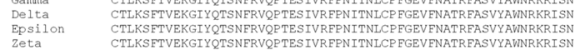

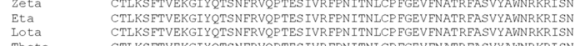

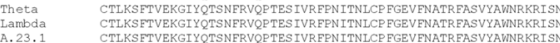

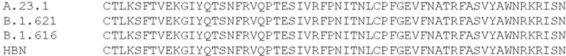

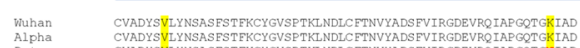

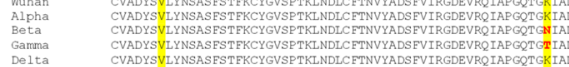

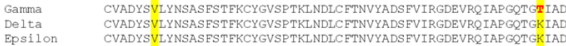

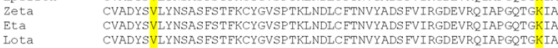

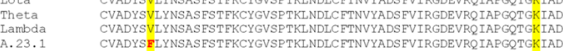

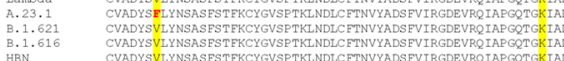

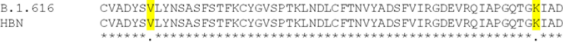

RBM

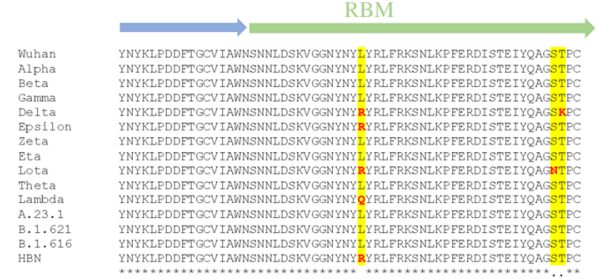

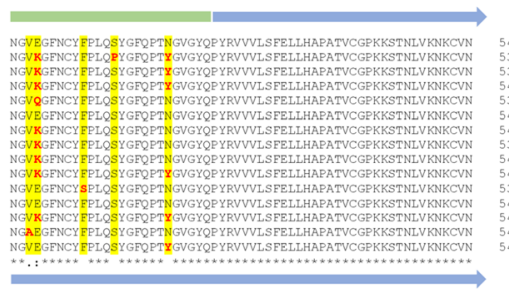

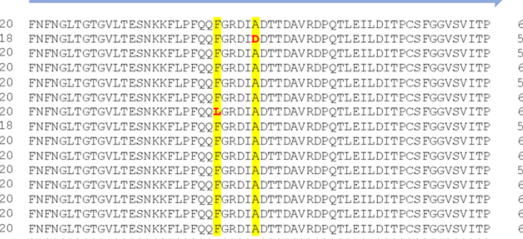

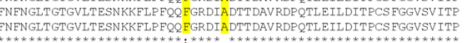
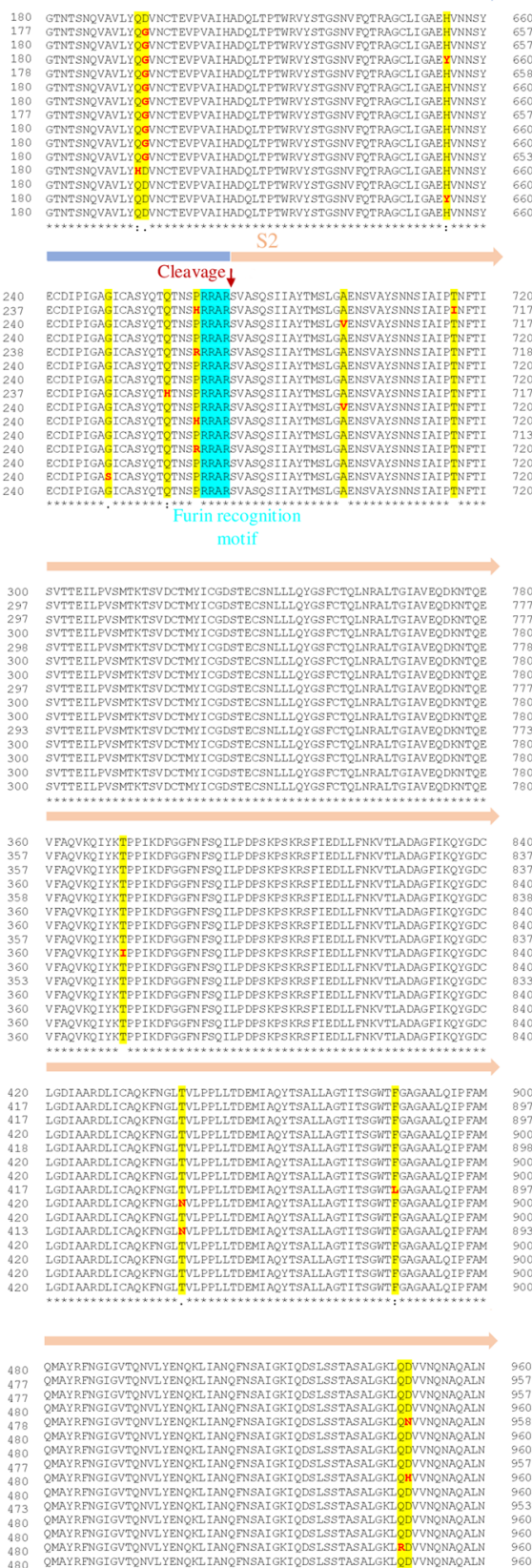
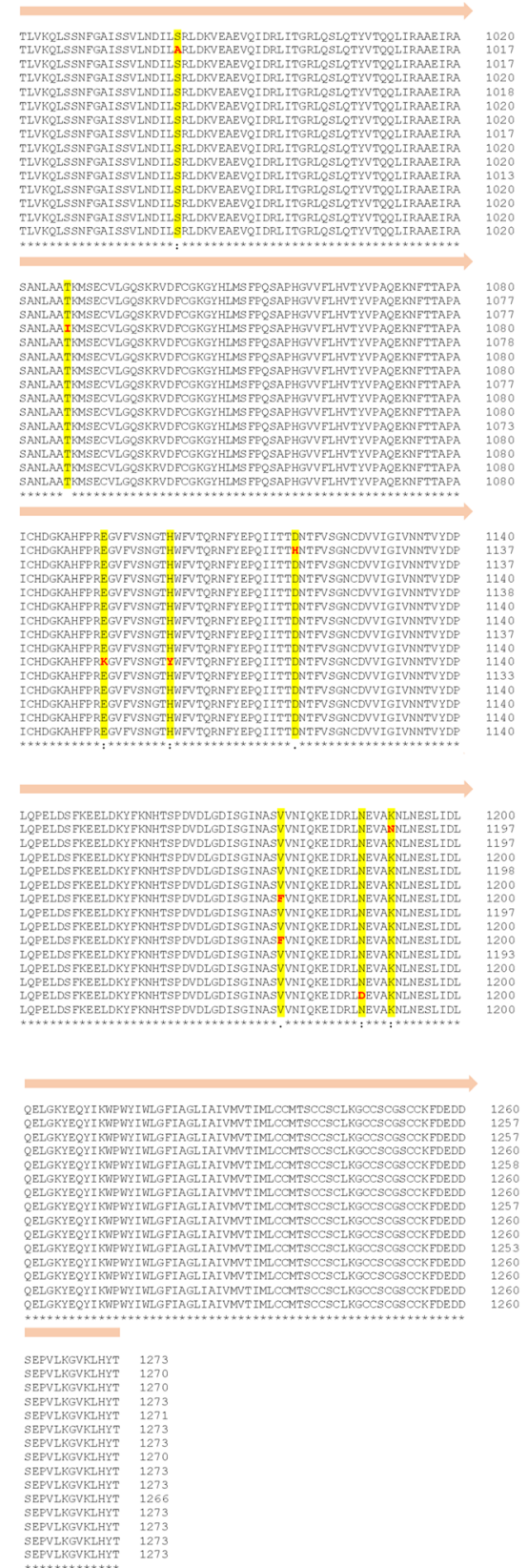

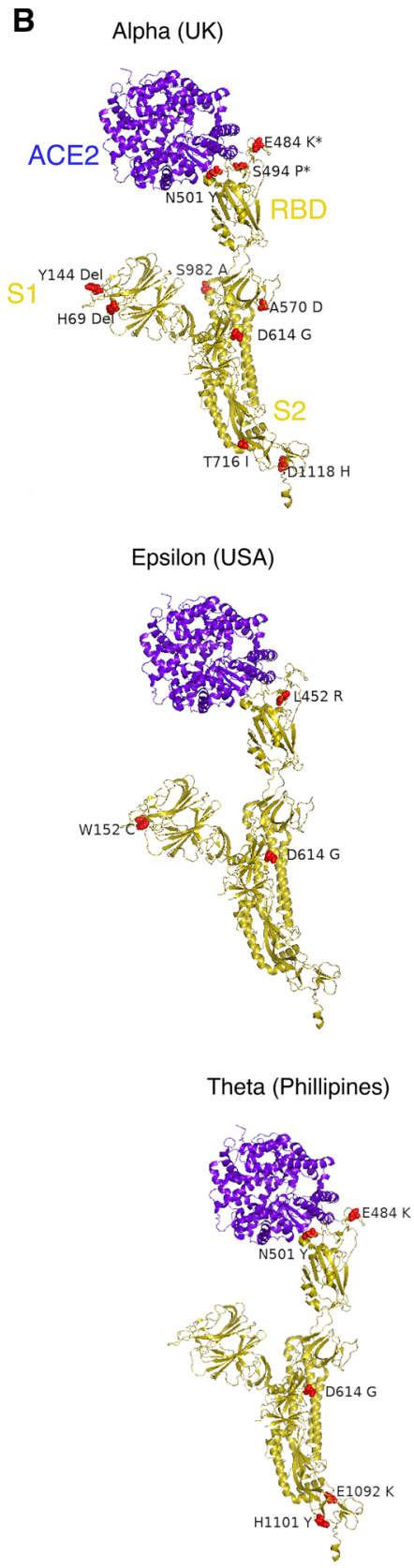

B.1.616(France)

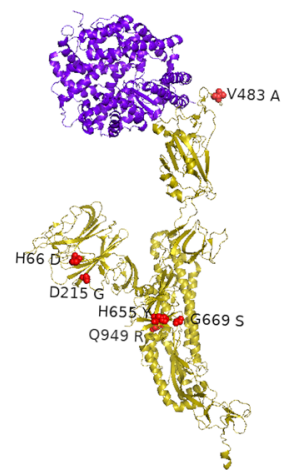

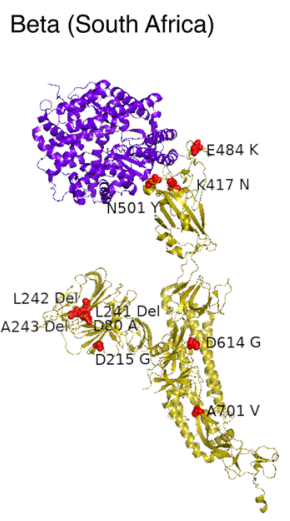
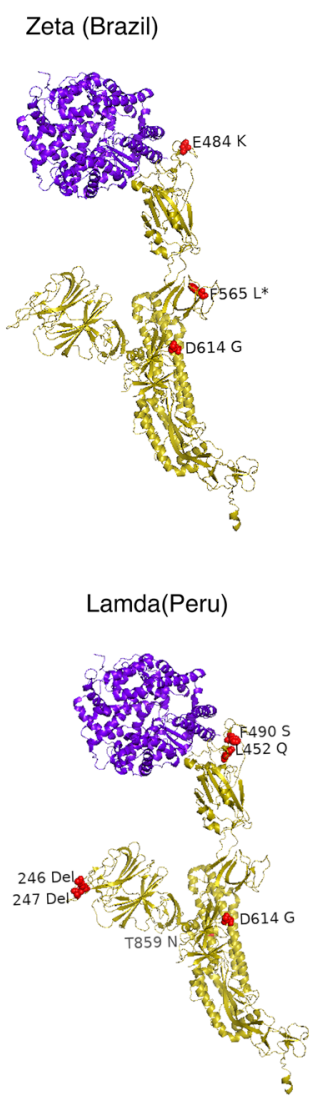

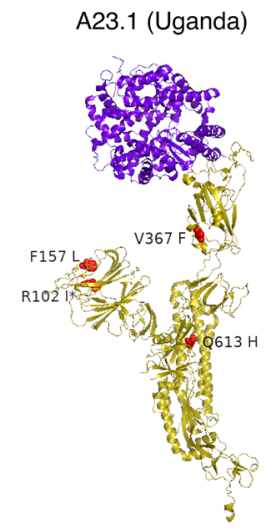

Gamma (Brazil)
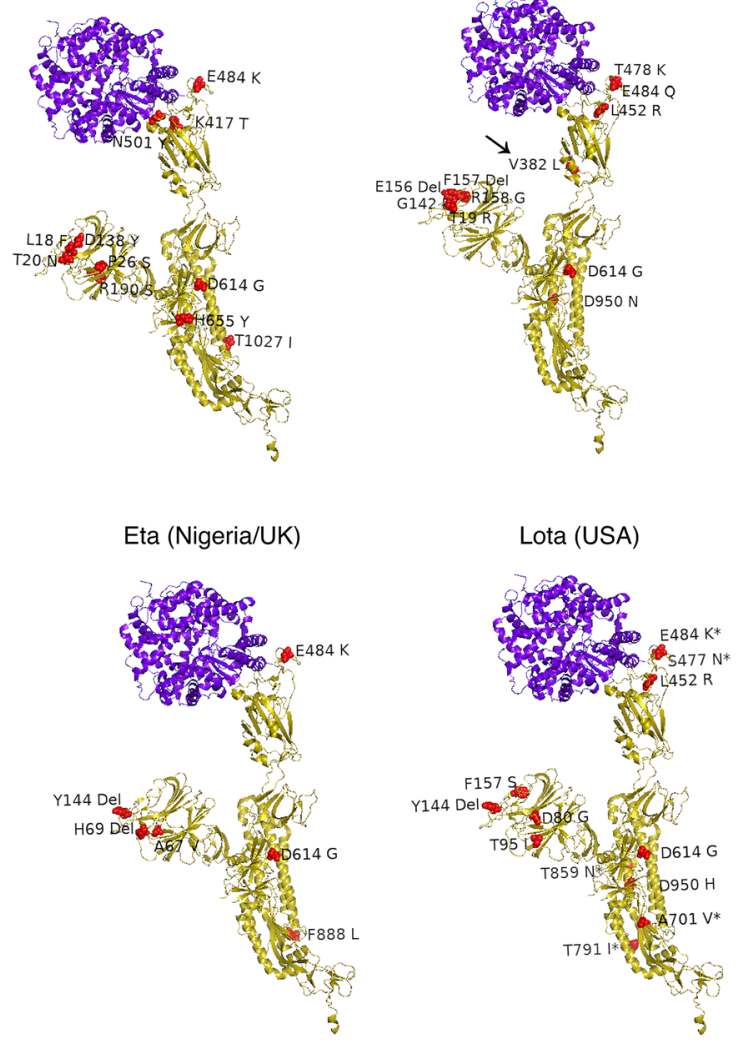

B.1.621(Columbia)

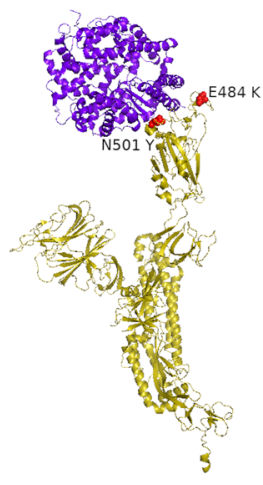

HMN.19B (France)

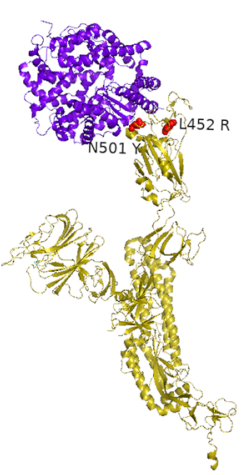

Figure 1. continued 
Covishield recipients have somewhat higher adverse effects as compared to Covaxin after immunization (46.7 vs 31.2\%) (Singh et al. 2021). Later, in order to ramp up the vaccination process, two new Indian vaccines from Zydus Cadila (ZyCoV-D) and Biological E have also entered the production phase.

\section{A hurdle to vaccination-emerging variants - Why so many variants?}

SARS-CoV-2 being a RNA virus belongs to the group of the fastest mutating pathogens in the world. The mutation rate in the Dengue virus is $2.64 \times$ $10^{-5}$ mutations per site per generation, while for HIV and Influenza it is $4 \times 10^{-5}$ and $1.35 \times 10^{-5}$ mutations per site per generation respectively. The exceptionally high mutation rates provide the RNA viruses with remarkable capabilities for adaptation to combat selection pressures. The main reason for the high mutation rates is the lack of proofreading activity in the reverse transcriptase enzymes of these viruses. However, the mutation rates are low in SARS CoV-1 $\left(9.0 \times 10^{-7}\right.$ mutations per nucleotide per replication cycle) and SARS CoV-2 $\left(1 \times 10^{-7}\right.$ substitutions/site/year). It was reported that coronavirus nsp14 acts as a $3^{\prime}-5^{\prime}$ exoribonuclease on both single-stranded and double-stranded RNA and reduces the mutational defects in the corona viruses (Carrasco et al. 2017).

Although the mutation rate of SARS-CoV-2 is not as high as HIV and Influenza, the situation of emerging variants through mutations has been aggravated by the extensive use of convalescent plasma therapy. In this therapy, plasma from recovered patients, termed as convalescent plasma, is used for the treatment of newly infected patients as it consists of neutralization antibodies. This therapy was successful in treating patients and reduced the severity of disease, which led to prevalent use of convalescent plasma among patients. However, it was observed that such mass usage of this therapy led to evolution of variants such as D796H substitution in $\mathrm{S} 2$ domain and deletion $\Delta \mathrm{H} 69 / \Delta 79$ in the $\mathrm{S} 1$ domain of the protein (Kemp et al. 2021). Importantly with the reduction of passively transferred antibodies, the dominant virus also diminished, suggesting that the virus is co-evolving based on the host immune responses. Further this double mutant virus showed less recognition with convalescent plasma but maintained the same infectivity rates (Collier et al. 2021).

Since most of the neutralization antibodies are against the $\mathrm{S}$ protein, this protein is under tremendous immune pressure from the host. It was observed that mutations in the $\mathrm{S}$ protein were responsible for the second and third waves of infection in most, if not all the countries. Notably, the second and third waves' peaks were higher with faster transmission rates and higher mortality among humans as compared to the first peak. From the sequencing of these viruses during the peak of infection, it was observed that a dominant mutation in the $\mathrm{S}$ protein is the cause of such infection rates and the virus is continuously evolving based on the immune pressure or demographics. For example, it was observed that, in India during the first wave, mostly older people with diabetes and cardiovascular disease were the vulnerable population. However, the second wave affected a considerable amount of people in the 25-45 age group; and the third wave is expected to affect children. Therefore, due to their continuous evolution, WHO is closely keeping record of all the mutations globally and classifying them as Variant of Concern (VOC) or variant of interest (VOI) based on their infection pattern. Mutants which have been recognized to cause community transmission/multiple COVID-19 cases/clusters, or have been detected in multiple countries are assessed to be a VOI by WHO in consultation with the WHO SARSCoV-2 Virus Evolution Working Group. Along with these characteristics if the virus poses one or more severe public health concerns like-increased transmissibility, virulence, change in the course of epidemiology, change in clinical presentation or evading the effectiveness of diagnostics, vaccines, therapeutics, it can be grouped under VOC. Below is the list of variants responsible for larger second and third waves of infection in many countries (table 2, figure 1).

\subsection{Alpha variant, B.1.1.7 (UK)}

This mutant virus was found to be more contagious than the wild type and harbors multiple mutations. The major mutations in the spike protein of B.1.1.7 were deletions 69-70, 144 in N terminal domain (NTD) and substitutions N501Y, A570D, D614G, P681H, T716I, S982A, D1118H in RBD and S1/S2 region of S protein (table 2, figure 1). However, some of the patients also had E484K, S494P, K1191N substitutions.

Further, this mutant showed decreased neutralization with BNT162b2 (Pfizer), or with antibodies from patients recovered from COVID-19. Moreover, the emergence of E484K in the B.1.1.7 background led to significant loss of neutralization activity as compared to the control Wuhan virus (Collier et al. 2021). 
112 Page 12 of 16

\subsection{Beta (South Africa), B.1.351}

The substitutions in this variant, D80A, D215G, K417N, E484K, N501Y, D614G, A701V and deletions in 241-243 (table 2; figure 1) emerged in a background of high HIV prevalence accompanied with high rates of population exposure (Tegally et al. 2020). The transmission was found to be 1.5 times higher for this variant where nearly $80 \%$ of the sequenced cases in Feb 2021 belonged to this mutant. In vitro data suggest that this variant may be able to establish infections in rats and mice (Yao et al. 2021), which seems to have been confirmed in vivo (Montagutelli et al. 2021).

Since most of the mutations were concentrated in the $\mathrm{RBM}$ and $\mathrm{S} 1$ region (figure $1 \mathrm{~B}$ ), as expected there was decreased neutralization of this mutant with convalescent plasma as well as with monoclonal antibodies (etesevimab, bamlanivimab, REGN10989) used for clearing the viral infection. Using pseudovirus that had all mutations of the Beta variant, it was observed the mRNA-1273 showed reduction in neutralization of this mutant by 6.4-fold (Wu et al. 2021). Similarly Astrazeneca vaccine did not confer any protection against this variant (Madhi et al. 2021). However, BNT162b2 from Pfizer was $100 \%$ effective against this variant (https://www.busin esswire.com/news/home/20210401005365/en/Pfizerand-BioNTech-Confirm-High-Efficacy-and-No-SeriousSafetyConcerns-Through-Up-to-Six-Months-FollowingSecond-Dose-in-UpdatedTopline-Analysis-of-LandmarkCOVID-19-Vaccine-Study).

\subsection{Gamma, P.1 (Brazil)}

This variant has been estimated to be 2.6 times more transmissible (Coutinho et al. 2021) and mostly consists of substitutions in the S1 region (L18F T20N, P26S, D138Y, R190S, K417T, D614G, H655Y, E484K, N501Y,T1027I; table 1 and figure 1B) and no deletion of amino acids were observed. Similar to the Beta variant, this one can also infect mice and rats (Yao et al. 2021; Montagutelli et al. 2021). The studies using convalescent plasma and BNT162b2 demonstrated reduced neutralization activity with this mutant strain (Garcia-Beltran et al. 2021).

\subsection{Delta, B.1.617 (India)}

This variant is considered a 'double mutant' virus as this is the only variant that has L452R and the E484Q substitutions in RBM domain of $\mathrm{S}$ protein. Both of these mutations are responsible for reduced recognition by neutralization antibodies mediating immune escape.

Based on the mutations in B1.617, it has been classified into three sub-lineages. B.1.617.1 possesses E484Q mutation, while B.1.617.2 lacks E484Q but contains D157,158 and T478K not present in the other two variants. B.1.617.3 has E484Q and D157,158 changes that can also be found in B.1.617.1. Recently in March 2021, substitution V382L was also found in around $15-20 \%$ of the sequenced viral genomes. Though it's too early to predict its effect on pathogenesis, this is the first time a substitution has been seen in this region that belongs to RBD but is not a part of RBM in S1 (https://www.pib.gov.in/Press releaseshare.aspx?PRID $=170717730$ march 2021; figure 1B). Although these sub lineages have different mutations, it is interesting to note that most of the mutations have occurred in the same S1 region, suggesting a selective pressure at this region in India (table 1 and figure 1).

Since B.1.617.2 was majorly responsible for the second wave of COVID-19 in India and because it got rapidly transferred to the UK as well, a study was conducted from 5 April to 16 May to analyze the effectiveness of two vaccine candidates- Pfizer and AstraZeneca against the B.1.617.2 variant compared to B.1.1.7. Based on the press release from Public Health England (PHE), both vaccines were $33 \%$ effective after 3 weeks of the second dose against the B.1.617.2 variant while it was $50 \%$ with B.1.1.7. But after 2 weeks of the second dose, Pfizer demonstrated effectiveness of $88 \%$ on the B.1.617.2 compared to $93 \%$ effectiveness against B.1.1.7. However, AstraZeneca did not show as major differences as Pfizer where it was $60 \%$ against B.1.617.2 and 66\% against B.1.1.7. (https://www. gov.uk/government/news/vaccines-highly-effective-agai nst-b-1-617-2-variant-after-2-doses).

Similarly, Covaxin conferred $65 \cdot 2 \%$ protection against the Delta variant. The trial reported no adverse effects and zero death due to vaccine administration (Ella et al. 2021a, b).

\subsection{Epsilon, B.1.429/427/Cal20-C (USA)}

This variant that originated in March 2020 was highly prevalent in southern California, USA and accounted for $50 \%$ of the sequenced viral genomes. This variant showed higher (18-24\%) transmission rates (Deng et al. 2021), but as compared to other mutants showed only 4 substitutions in the $\mathrm{S}$ protein with no deletion of amino acids. 


\subsection{Zeta, P.2 (Brazil)}

This variant originated in Brazil but was detected before the Gamma variant and has only 4 substitutions where D614G and $\mathrm{E} 484 \mathrm{~K}$ are the common substitutions that were also found in the Gamma variant. Similarly this variant is linked to reductions in neutralizing antibody titres of convalescent plasma or vaccine sera (Garcia-Beltran et al. 2021). A recent study from ICMR estimated the efficacy of Covaxin against this variant and found that a reduction of 1.09 and 1.92 fold in neutralization titres with sera from individuals with natural infection from this variant or from Covaxin vaccine recipients (Sapkal et al. 2021).

\subsection{Eta, B.1.525 (Nigeria/UK)}

This variant has been reported in several countries at low frequency, and possesses deletions in spike protein- $\Delta \mathrm{H} 69-\mathrm{V} 70$ and $\Delta 144$, and substitutions E484K, Q677H and F888L. The independent emergence of $\mathrm{Q} 677 \mathrm{H}$ in this variant and in some USA variants provides strong evidence of adaptation, potentially through an effect of this mutation on the proximal polybasic furin cleavage site, although exact effect of this mutation is yet unclear (Harvey et al. 2021).

\subsection{Lota, B.1.526 (USA)}

This variant that originated in November 2020 showed increased prevalence in the North-East USA and was associated with patients being more frequently hospitalized (Annavajhala et al. 2021). Currently, this mutant virus exhibits the maximum number of changes that is in contrast to the Epsilon variant that originated in the USA in March 2020. Importantly the mutations were found to be present throughout the ordered structure of the protein (figure 1B), indicating that the virus is evolving very fast to combat immune pressure from the host. The Epsilon virus with D614G background has two sub-variants; E484K and S477N mutations. The studies with convalescent sera and vaccine-elicited antibodies from the USA showed that $\mathrm{S} 477 \mathrm{~N}$ mutant is completely neutralized while a 3.5-fold decrease was observed with E484K variant compared to D614G as control (Zhou et al. 2021).

\subsection{Theta, P.3, Philippines}

This variant notably contains the E484K, N501Y and D614G mutations that are found in the three variants classified as VOCs by the WHO (Alpha, Beta and Gamma), which have been linked to increased ACE2 affinity/transmissibility, ability to use the ACE2 in rats and mice, as well as decreased effectiveness of monoclonal antibody, etesevimab (Yao et al. 2021; Wang et al. 2021).

\subsection{Lambda, C.37, Peru}

This variant has substitutions in L452Q, D614G F490S, T859N, G75V, T76I, and deletion from 246-252. However, no data is available on the effect on its neutralization with convalescent sera and vaccine elicited antibodies.

\subsection{A.23.1, Uganda}

This variant is notable for its lack of the D614G mutation, with a Q613H mutation instead that may be functionally similar. Apart from this, it has substitutions in F157L,V367F, P681R, R102I* (table 2). The substitution at F157L was found to reduce the neutralizing ability of the mAb CoV2-2489 against this variant (Harvey et al. 2021). It replaced previously circulating viruses in Uganda within 2 months, suggesting higher transmissibility but its clinical impact is still not clear (Bugembe et al. 2021).

\subsection{B.1.621, Columbia}

This is a recently evolved variant with mutation in E484K and N501Y.

\subsection{B.1.616, France}

This variant emerged during a nosocomial outbreak in a geriatric ward with substitutions- H66D, D215G, V483A, H655Y, G669S, Q949R and N1187D (table 2). Many cases were severe and most patients were above 81 years of age. This variant is notable for yielding weaker RT-PCR positives, along with a lower detection rate from nasopharyngeal samples, indicating the possibility of altered tropism. The infection was mostly localized to Brittany, France. Transmission beyond Brittany therefore seems limited, but notably it does possess a mutation linked to transmission in felines (Braun et al. 2021).

\subsection{HMN.19B Henri Mondor, France}

This variant was first detected in immunocompromised patients and contains the L452R and N501Y mutations, 
both of which were found to be linked to increased transmissibility. This variant is from clade 19B, that had become rare from early 2020 and lacks the common D614G mutation; thus its resurgence in this variant may indicate that these mutations confer increased transmissibility (Fourati et al. 2021; Volz et al. 2021).

\section{Conclusion}

Although variants showed different mutations that would be dependent on the general immunity of the population or demographics of countries, we observed that some mutations in $\mathrm{S}$ protein were common irrespective of their origin, indicating that these mutants might provide the selective advantage to the virus. One of the most common mutations is D614G that was observed in almost all the variants. Using in vitro infection studies with the mutated virus, this substitution was either associated with enhanced viral replication, stability (Plante et al. 2021) or decreased S1 shedding and higher density of S protein in the virion (Zhang et al. 2020). The virus with N501Y substitution possessed higher affinity towards ACE2 that led to better infection in humans. Similarly, the substitution of E484 to either K or Q was associated with increased ACE2 affinity and in addition was also responsible for immune escape. Another common variant is L452R that is linked to transmissibility or immune escape from a monoclonal antibody, bamlanivimab used for COVID-19 treatment (Starr et al. 2021). Besides substitutions, deletions of amino acids were also observed and deletion in two aminoacids $(\Delta 69$ and $\Delta 70)$ in NTD domain of S1 is responsible for increased infectivity rates and decreased serum neutralization (McCarthy et al. 2021; Kemp et al. 2021). In contrast, substitution of $\mathrm{K} 417$ to either $\mathrm{N}$ or $\mathrm{T}$ substitutions in some patients led to moderate decrease in ACE2 binding affinity but that was somehow compensated by N501Y substitution (Boehm et al. 2021).

Combining these observations together, the virus is mostly preparing itself to increase its affinity towards ACE2 receptor or immune escape. Therefore, the next generation of candidate antigen(s) should be able to accommodate at least these mutations, in order to elicit antibodies that can neutralize the virus effectively. It has been suggested that the use of combinatorial antigen by Heisch et al. could provide an enhanced immune response. Currently, another alternative is to vaccinate with a mixture of vaccines. However, this needs to be considered in regards to safety and side effects shown by various vaccines. Further the above strategies would only lead to stronger immune response against the wild-type $\mathrm{S}$ protein rather than providing neutralization of the variants that have changes in the sequence of $\mathrm{S}$ protein. Therefore, booster vaccination doses with $\mathrm{S}$ protein containing major prevalent mutations could be a feasible strategy to effectively provide immunity to the population against mutant viruses.

\section{Acknowledgements}

The authors would like to thank IISER Tirupati, DSTSERB for funding. SG is supported by IISER Tirupati, DST SERB (Grant No. ECR/2017/001703), BD by DSTINSPIRE fellowship and RV by IISER Tirupati fellowship.

\section{References}

Andersen KG, Rambaut A, Lipkin WI, Holmes EC and Garry RF 2020 The proximal origin of SARS-CoV-2. Nat. Med. 26 450-452

Annavajhala MK, Mohri H, Wang P, et al. 2021 A novel SARS-CoV-2 variant of concern, B. 1.526, identified in New York. medRxiv

Blood Clotting following COVID-19 Vaccination: Information for Health Professionals https://www.publichealth. hscni.net/publications/blood-clotting-following-covid-19vaccination-information-health-professionals

Boehm E, Kronig I, Neher RA, Eckerle I, Vetter P and Kaiser L 2021 Novel SARS-CoV-2 variants: the pandemics within the pandemic. Clin. Microbiol. Infec. 27 1109-1117

Braun KM, Moreno GK, Halfmann PJ, et al. 2021 Transmission of SARS-CoV-2 in domestic cats imposes a narrow bottleneck. PLoS Pathog. 17 e009373

Bugembe DL, Phan MV, Ssewanyana I, et al. 2021 A SARS-CoV-2 lineage A variant (A. 23.1) with altered spike has emerged and is dominating the current Uganda epidemic. Nat. Microbiol. 6 1094-1101

Carrasco HR, Jácome R, López VY and de Ponce LS 2017 Are RNA viruses candidate agents for the next global pandemic? A review. ILAR J. 58 343-358

Collier DA, De Marco A, Ferreira IA, et al. 2021 Sensitivity of SARS-CoV-2 B. 1. 1. 7 to mRNA vaccine-elicited antibodies. Nature 593 136-141

Coutinho RM, Marquitti FMD, Ferreira LS, et al. 2021 Model-based estimation of transmissibility and reinfection of SARS-CoV-2 P. 1 variant. medRxiv

COVID-19 vaccine tracker and landscape https://www.who. int/publications/m/item/draft-landscape-of-covid-19-candidatevaccines

Dagan N, Barda N, Kepten E, et al. 2021 BNT162b2 mRNA Covid-19 vaccine in a nationwide mass vaccination setting. N. Engl. J. Med. 384 1412-1423 
Deb B, Shah H and Goel S 2020 Current global vaccine and drug efforts against COVID-19: Pros and cons of bypassing animal trials. J. Biosci. $451-10$

Deng X, Garcia-Knight MA, Khalid MM, et al. 2021 Transmission, infectivity, and antibody neutralization of an emerging SARS-CoV-2 variant in California carrying a L452R spike protein mutation. MedRxiv

Ebinger JE, Fert-Bober J, Printsev I, et al. 2021 Antibody responses to the BNT162b2 mRNA vaccine in individuals previously infected with SARS-CoV-2. Nat. Med. 27 981-984

Ella R, Reddy S, Blackwelder W, et al. 2021a Efficacy, safety, and lot to lot immunogenicity of an inactivated SARS-CoV-2 vaccine (BBV152): a double-blind, randomised, controlled phase 3 trial. medRxiv

Ella R, Vadrevu KM, Jogdand H, Prasad S, Reddy S, Sarangi V, Ganneru B, Sapkal G, et al. 2021b Safety and immunogenicity of an inactivated SARS-CoV-2 vaccine, BBV152: a double-blind, randomised, phase 1 trial. Lancet Infect. Dis. 21 637-646

Fourati S, Decousser JW, Khouider S, et al. 2021 Novel SARS-CoV-2 variant derived from clade 19B, Frace. Emerg. Infect. Dis. 271540

Ganneru B, Jogdand H, Daram VK, et al. 2021 Th1 skewed immune response of whole virion inactivated SARS CoV 2 vaccine and its safety evaluation. Science 24102298

Garcia-Beltran WF, Lam EC, Denis KS, Nitido AD, Garcia ZH, Hauser BM and Balazs AB 2021 Multiple SARS$\mathrm{CoV}-2$ variants escape neutralization by vaccine-induced humoral immunity. Cell 184 2372-2383

Genome Sequencing by INSACOG shows variants of concern and a novel variant in India http://www.pib.gov. in/Pressreleaseshare.aspx? $P R I D=1707177$

Gralinski LE and Menachery VD 2020 Return of the Coronavirus: 2019-nCoV. Viruses 12 135-141

Harvey WT, Carabelli AM, Jackson B, Gupta RK, Thomson EC, Harrison EM and Robertson DL 2021 SARS-CoV-2 variants, spike mutations and immune escape. Nat. Rev. Microbiol. 19 409-424

Hsieh CL, Goldsmith JA, Schaub JM, et al. 2020 Structurebased design of prefusion-stabilized SARS-CoV-2 spikes. Science 369 1501-1505

Huang Y, Yang C, Xu XF, Xu W and Liu SW 2020 Structural and functional properties of SARS-CoV-2 spike protein: potential antivirus drug development for COVID-19. Acta Pharmacol. Sin. 41 11411149

Kemp SA, Collier DA, Datir RP, Ferreira IA, Gayed S, Jahun A, Hosmillo M, Rees-Spear C, et al. 2021 SARS-CoV-2 evolution during treatment of chronic infection. Nature $\mathbf{5 9 2}$ 277-282

Kim D, Lee JY, Yang JS, Kim JW, Kim VN and Chang H 2020 The architecture of SARS-CoV-2 transcriptome. Cell 181 914-921
Lan J, Ge J, Yu J, et al. 2020 Structure of the SARS-CoV-2 spike receptor-binding domain bound to the ACE2 receptor. Nature $\mathbf{5 8 1} 215-220$

Madhi SA, Baillie V, Cutland CL, Voysey M, Koen AL, Fairlie L and Izu A 2021 Efficacy of the ChAdOx1 nCoV19 Covid-19 vaccine against the B. 1.351 variant. New Engl. J. Med. 384 1885-1898

Marshall M, Ferguson ID, Lewis P, Jaggi P, Gagliardo C, Collins JS, et al. 2021 Symptomatic acute myocarditis in seven adolescents following Pfizer-BioNTech COVID-19 vaccination. Pediatrics 148 e2021052478

McCarthy KR, Rennick LJ, Nambulli S, Robinson-McCarthy LR, Bain WG, Haidar G and Duprex WP 2021 Recurrent deletions in the SARS-CoV-2 spike glycoprotein drive antibody escape. Science 371 1139-1142

Montagutelli X, Prot M, Levillayer L, et al. 2021 The B1. 351 and P. 1 variants extend SARS-CoV-2 host range to mice. BioRxiv

Naaber P, Adamson A, Sepp E, Tserel L, Kisand K and Peterson P 2021 Antibody response after COVID-19 mRNA vaccination in relation to age, sex, and side effects. medRxiv

Olliaro P, Torreele E and Vaillant M 2021 COVID-19 vaccine efficacy and effectiveness - the elephant (not) in the room. Lancet Microbe 2 E279-E280

Pallesen J, Wang N, Corbett KS, Wrapp D, Kirchdoerfer RN, Turner HL, Cottrell CA, Becker MM, et al. 2017 Immunogenicity and structures of a rationally designed prefusion MERS-CoV spike antigen. Proc. Nat. Acad. of Sci. 114 E7348-E7357

Pfizer and BioNTech Confirm High Efficacy and No Serious Safety Concerns Through Up to Six Months Following Second Dose in Updated Topline Analysis of Landmark COVID-19 Vaccine Study [Internet]. 2021 [cited 2021 Apr 1]. Available from: https://www.businesswire.com/ news/home/20210401005365/en/Pfizer-and-BioNTechConfirm-High-Efficacy-and-No-Serious-SafetyConcernsThrough-Up-to-Six-Months-Following-Second-Dose-inUpdatedTopline-Analysis-of-Landmark-COVID-19-VaccineStudy

Plante JA, Liu Y, Liu J, et al. 2021 Spike mutation D614G alters SARS-CoV-2 fitness. Nature 592 116-121

Sadoff J, Davis K and Douoguih M 2021 Thrombotic thrombocytopenia after Ad26. COV2. S vaccinationresponse from the manufacturer. New Engl. J. Med. 384 1965-1966

Sapkal G, Yadav PD, Ella R, et al. 2021 Neutralization of VUI B. 1.1. 28 P2 variant with sera of COVID-19 recovered cases and recipients of Covaxin an inactivated COVID-19 vaccine. J. Travel Med. 28 taab077

Sars-CoV-2 circulating variants https://viralzone.expasy.org/ variants

SARS-CoV-2 Variant Classifications and Definition https:// www.cdc.gov/coronavirus/2019-ncov/variants/variant-info. html 
Singh AK, Jena A, Kumar MP, Sharma V and Sebastian S 2021 Risk and outcomes of coronavirus disease in patients with inflammatory bowel disease: A systematic review and meta-analysis. United Eur. Gastroenterol. J. 9 159-176

Starr TN, Greaney AJ, Dingens AS and Bloom JD 2021 Complete map of SARS-CoV-2 RBD mutations that escape the monoclonal antibody LY-CoV555 and its cocktail with LY-CoV016. Cell Rep. Med. 2100255

Status of COVID-19 Vaccines within WHO EUL/PQ evaluation process (WHO) https://extranet.who.int/

Tegally H, Wilkinson E, Giovanetti M, et al. 2020 Emergence and rapid spread of a new severe acute respiratory syndrome-related coronavirus 2 (SARS-CoV-2) lineage with multiple spike mutations in South Africa. MedRxiv

The Sinopharm COVID-19 vaccine: What you need to know https://www.who.int/news-room/feature-stories/detail

The Sinovac COVID-19 vaccine: What you need to know https://www.who.int/news-room/feature-stories/detail

Tracking-SARS-CoV-2-variants https://www.who.int/en/ activities/

Vaccines-highly-effective-against-b-1-617-2-variant-after-2doses- hcighly-effective-against-b-1-617-2-variant-after2-doses

Volz E, Hill V, McCrone JT, et al. 2021 Evaluating the effects of SARS-CoV-2 spike mutation D614G on transmissibility and pathogenicity. Cell $\mathbf{1 8 4}$ 64-75

Wang H, Zhang Y, Huang B, et al. 2020 Development of an inactivated vaccine candidate, BBIBP-CorV, with potent protection against SARS-CoV-2. Cell 182 713-721
Wang P, Nair MS, Liu L, et al. 2021 Antibody resistance of SARS-CoV-2 variants B. 1.351 and B. 1.1. 7. Nature 593 130-135

Wrapp D, Wang N, Corbett KS, Goldsmith JA, Hsieh CL, Abiona O, Graham BS and McLellan JS 2020 Cryo-EM structure of the 2019-nCoV spike in the prefusion conformation. Science 367 1260-1263

Wu K, Werner AP, Moliva JI, et al. 2021 mRNA-1273 vaccine induces neutralizing antibodies against spike mutants from global SARS-CoV-2 variants. BioRxiv

Xu C, Wang Y, Liu C, Zhang C, Han W, Hong X, Wang Y, Hong Q, et al. 2021 Conformational dynamics of SARS$\mathrm{CoV}-2$ trimeric spike glycoprotein in complex with receptor ACE2 revealed by cryo-EM. Sci. Adv. 7 eabe5575

Yao W, Wang Y, Ma D, et al. 2021 Circulating SARS-CoV-2 variants B. 1.1. 7, 501Y. V2, and P. 1 have gained ability to utilize rat and mouse Ace2 and altered in vitro sensitivity to neutralizing antibodies and ACE2-Ig. bioRxiv

Zhang L, Jackson CB, Mou H, Ojha A, Peng H, Quinlan BD, Rangaraja ES, Pan A, et al. 2020 SARS-CoV-2 spike-protein D614G mutation increases virion spike density and infectivity. Nat. Commun. 11 1-9

Zhou P, Yang XL, Wang XG, et al. 2020 A pneumonia outbreak associated with a new coronavirus of probable bat origin. Nature $579270-273$

Zhou H, Dcosta BM, Samanovic MI, Mulligan MJ, Landau NR and Tada T 2021 B. 1.526 SARS-CoV-2 variants identified in New York City are neutralized by vaccineelicited and therapeutic monoclonal antibodies. mBio $\mathbf{1 2}$ e0138621

Corresponding editor: BJ RAO 